\title{
电极材料调控产物选择性
}

\author{
陈 娜 ${ }^{a} \quad$ 赖小丽 $b \quad$ 徐海超 ${ }^{*}, b$ \\ ( ${ }^{a}$ 华侨大学生物医学学院 厦门 361021) \\ ${ }^{b}$ 厦门大学化学化工学院 福建省化学生物学重点实验室 厦门 361005)
}

\section{Electrode Materials Tune Product Selectivity}

\author{
Chen, $\mathrm{Na} \quad$ Lai, Xiaoli $\quad \mathrm{Xu}$, Haichao* \\ ( ${ }^{a}$ School of Biomedical Sciences, Huaqiao University, Xiamen 361021) \\ $\left({ }^{b}\right.$ Key Laboratory of Chemical Biology of Fujian Province, College of Chemistry and Chemical Engineering, \\ Xiamen University, Xiamen 361005)
}

有机电合成利用电能驱动有机合成反应, 可避免使 用氧化还原试剂, 是一种绿色的合成工具. 由于电极的 氧化还原能力连续可调, 电化学为控制有机合成反应活 性和选择性提供便利的工具. 在有机电合成中, 电极表 面对反应物或中间体的吸附可影响电子转移及后续反 应的热力学和动力学, 进而影响整个转化的选择性. 如 徐海超课题组利用氟嗍酸盐在阳极表面的吸附富集发 展了高选择性的烯烃双官能团化反应 ${ }^{[1]}$. 该课题组还通 过改变阴极材料实现了联芳基酮肜环化反应的产物选 择性调控(Scheme 1a) ${ }^{[2]}$. 当使用析氢超电势较低的 Pt 基 阴极时，阴极发生析氢反应，联芳基酮肟发生阳极氧化 促进的脱氢环化得到氮芳杂环氮氧化物. 而当使用 $\mathrm{Pb}$ 阴极时, 由于其析氢超电势较高, 阳极氧化形成的氮芳 杂环氮氧化物优先在阴极被还原得到氮芳杂环化合物. 电极材料也可影响单电子和两电子氧化之间的选择性. 如羧酸经阳极氧化脱酸得到碳自由基, 使用铂阳极时, 碳自由基倾向于二聚从而得到单电子氧化产物, 而使用 碳阳极时, 由于碳电极对碳自由基中间体的吸附导致其 易发生进一步的电子转移形成碳正离子, 从而实现两电 子氧化 ${ }^{[3]}$.

2019 年徐海超课题组报道了二级芳胺的电化学氧 化二聚反应(Scheme 1b) ${ }^{[4]}$. 最近陈建宾课题组巧妙地利 用电化学反应的灵活可调性, 通过使用不同电极材料实 现了氨基吡啶的选择性单电子氧化二聚和两电子氧化 环化反应(Scheme 1c). 反应设计主要考虑利用电极材料
以及电流密度控制反应活性中间体的形成速率及其转 化路径实现反应选择性调控.

作者发现, 使用 $\mathrm{Pt}$ 片为阳极, 四丁基碘化胺为媒介 和电解质时, $N$-芳基氨基吡啶发生二聚反应得到四芳基 肼, 而使用石墨为阳极, 在弱碱性水溶液中电解则经两 电子氧化得到了环化产物. 对于环化反应, 使用其它的 碳材料作阳极如碳纤维、碳布、网状玻璃碳(RVC)反应 产率较低 $(<20 \%)$, 而使用 $\mathrm{Pt}$ 阳极则基本没有环化产物 生产 $(<5 \%)$. 作者对于环化产物的形成机制进行了研 究, 排除了四芳基肼作为反应中间体的可能.

作者根据机理实验和密度泛函理论计算提出了反 应的可能途径(Scheme 2). 由于碘离子的氧化电势比氨 基吡啶低(Scheme 2a), 其在阳极氧化生成 $\mathrm{I}_{2}$, 随后与氨 基吡啶反应形成 $\mathrm{N}-\mathrm{I}$ 化合物. $\mathrm{N}-\mathrm{I}$ 键发生均裂得到氮 自由基 ${ }^{[6-8]}$, 后者二聚得到四芳基肼产物. 在直接电解条 件下, 氨基吡啶在石墨阳极发生单电子氧化并失去质子 得到氮自由基(Scheme $2 b$ ). 除电流密度这一电化学反 应控制因子之外，其他的影响因子如石墨阳极比其它电 极材料如 Pt 具有更多的顺磁中心能使氮自由基吸附于 表面从而促使进一步的电子转移反应得到两电子氧化 的环化产物 ${ }^{[3]}$.

综上所述，陈建宾课题组创造性地利用不同电解条 件实现了氨基吡啶的选择性转化反应, 不仅为咪唑并 $[1,2-a]$ 吡啶提供高效绿色的合成方法，也为反应选择性 调控提供了新的思路, 将启发更多的相关研究.

\footnotetext{
* Corresponding author. E-mail: haichao.xu@xmu.edu.cn. Published online June 25, 2020.
} 
(a) Divergent electrochemical cyclization of ketoximes



b) Electrochemical dehydrogenative dimerization of secondary aryl amines

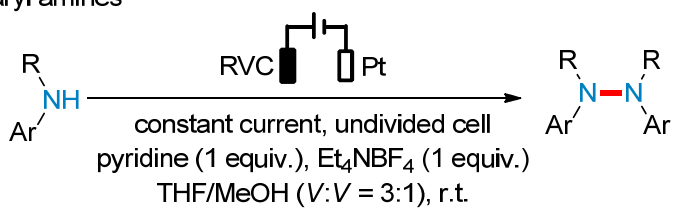

c) Divergent electrochemical oxidation of secondary aryl amines (Chen and coworkers)

图式 1 电极材料调控产物选择性

Scheme 1 Electrode materials determine product selectivity

a) Plausible mechanism for dimerization

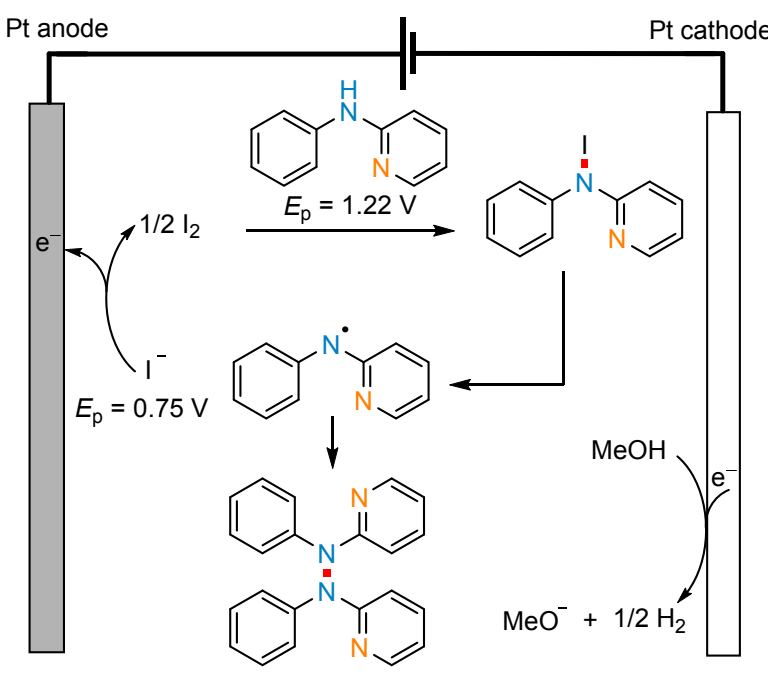

b) Plausible mechanism for cyclization

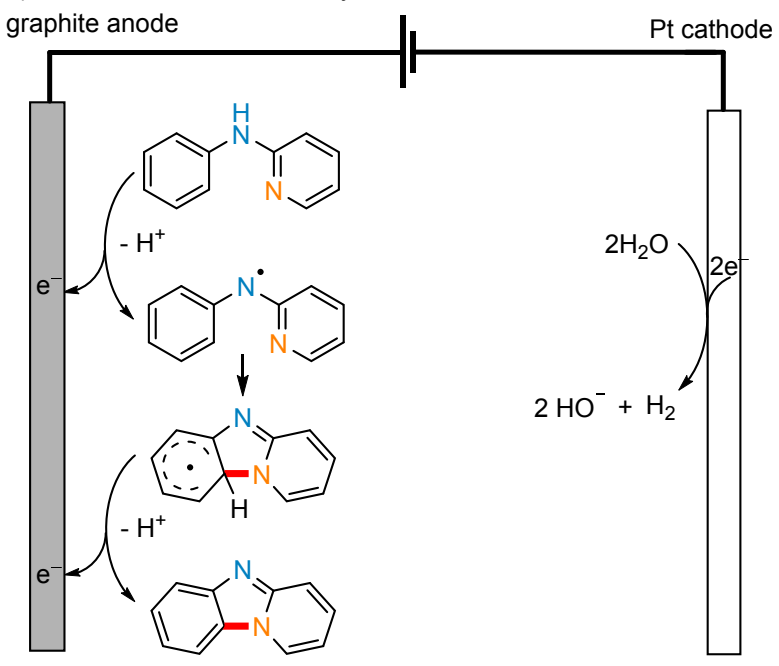

图式 2 可能反应机理

Scheme 2 Plausible mechanisms

\section{References}

[1] Xiong, P.; Long, H.; Song, J.; Wang, Y.; Li, J.-F.; Xu, H.-C. J. Am. Chem. Soc. 2018, 140, 16387.

[2] Zhao, H.-B.; Xu, P.; Song, J.; Xu, H.-C. Angew. Chem. Int. Ed. 2018, $57,15153$.

[3] Ross, S. D.; Finkelstein, M. J. Org. Chem. 1969, 34, 2923.

[4] Feng, E.-Q.; Hou, Z.-W.; Xu, H.-C. Chin. J. Org. Chem. 2019, 39, 1424 (in Chinese).
(冯恩祺, 侯中伟, 徐海超, 有机化学, 2019, 39, 1424.)

[5] Lv, S.; Han, X.; Wang, J. Y.; Zhou, M.; Wu, Y.; Ma, L.; Niu, L.; Gao, W.; Zhou, J.; Hu, W.; Cui, Y.; Chen, J. Angew. Chem. Int. Ed. 2020, $59,11583$.

[6] Xiong, P.; Xu, H. C. Acc. Chem. Res. 2019, 52, 3339.

[7] Hou, Z.-W.; Yan, H.; Song, J.-S.; Xu, H.-C. Chin. J. Chem. 2018, $36,909$.

[8] Hou, Z.-W.; Xu, H.-C. Chin. J. Chem. 2020, $38,394$. 